\title{
Channel Allocation in Dense Wavelength Division Multiplexing Radio-over-Fiber Networks
}

\author{
Mirosław Klinkowski*, Marek Jaworski*, and Davide Careglio ${ }^{\dagger}$ \\ * Z-14, National Institute of Telecommunications, 51-501 Wroctaw, Poland, mklinkow@itl.waw.pl \\ † CCABA, Universitat Politècnica de Catalunya, 08034 Barcelona, Spain
}

\begin{abstract}
In this paper we address the problem of the Radio-over-Fiber (RoF) network design. In particular, we focus on a DWDM RoF network for which we formulate a maximal service RoF channel allocation (MSCA) problem. Although MSCA is a difficult problem, still it can be resolved for the network instances of some hundred of access points and users.
\end{abstract}

Keywords: DWDM, Network Design, Radio-over-Fibre, Resources Allocation.

\section{INTRODUCTION}

The integration of wired and wireless networks has recently gained considerable research interest. The FiberWireless (FiWi) architectures [1] are very attractive since they combine the best attributes of both worlds. The optical networks offer almost limitless amount of bandwidth and wireless networks provide ubiquitous access and support user mobility.

One of the promising FiWi solutions is the Radio-over-Fiber (RoF) technology [2]. RoF networks allow an optical link to transmit a modulated radio frequency (RF) signal, by these means, moving the processing functionalities to the Central Station (CS) and reducing the radio Access Point (AP) complexity. In some deployment scenarios, such as e.g. picocellurar access networks, hundreds of antennas need to be supported and the RoF approach can result in a simplified overall system design due to the aggregation of RF signal generation and network management at a central location [3].

At the front end of the RoF network, millimeter-wave (mm-wave) or higher frequency are potentially useful RF resources to cope with $\mathrm{Gb} / \mathrm{s}$ data transmission [3]. A passive optical network $(\mathrm{PON})$ is a short-term candidate for the optical back end of the RoF network. The wavelength division multiplexed PON (WDM-PON) is considered to be the next evolutionary solution which increases system capacity and scalability as well as it improves network security [4]. The introduction of wavelength routing capabilities [5] will allow the reconfigurable DWDM-RoF networks to accommodate a huge number of APs with a flexible allocation of resources according to traffic demands and the movement of nomadic users.

Similarly as in DWDM core networks, the problem of channel allocation appears in reconfigurable DWDMRoF access networks [6]. Indeed, the RoF resources, such as the optical frequencies in the optical part of the network and RFs in the radio part of the network, have to be assigned appropriately to the APs so that to provide the users with the demanded bandwidth. At the same time, the channel interference between adjacent APs should be avoided in the RF domain. To the best of our knowledge, the RoF channel allocation problem in reconfigurable DWDM-RoF networks has not been studied in the literature so far. In this paper, as a case study, we focus on the problem of RoF channel allocation with maximal service objective (MSCA), which is a variant of the well-known frequency assignment problem (FAP) in wireless networks [8]. We provide a 0-1 integer programming (IP) formulation of MSCA and present some numerical results which enable further discussion on the problem complexity.

The reminder of this paper is organized as follows. In Section 2 we present the details of the considered DWDM RoF network scenario. In Section 3 we formulate the MSCA problem. In Section 4 we present some numerical results. Finally, in Section 5 we conclude obtained results.

\section{DWDM ROF NETWORK}

In this paper we focus on a reconfigurable DWDM RoF access network presented in Fig. 1. The network consists of a Central Station (CS) and a number of subnetworks, each one identified with a Remote Access Node (RAN) and a number of Access Points (AP). In the network, the connectivity between CS with RANs and between each RAN with its APs is achieved by means of optical fiber links.

CS is responsible for the RF signal processing and management of subnetworks. In the reminder of this paper, we assume each subnetwork applies the DWDM RoF technology developed by J.J. Vegas Olmos et al in [6]. In particular, the CS-RAN fiber link carries a number of RoF channels, each one corresponding to an optical carrier frequency signal modulated by an RF signal [6]. In this paper, we assume that the RF signals carried in the same fiber are orthogonal (i.e., they do not interfere in the air) and, hence, there is no interference between APs belonging to the same subnetwork. In general, there is no general rule for the RF generation and assignment to the RoF channels. Indeed, the DWDM RoF technology assures the separation of carrier frequencies, and thus unrestricted transmission of RF signals in the optical fiber link. 


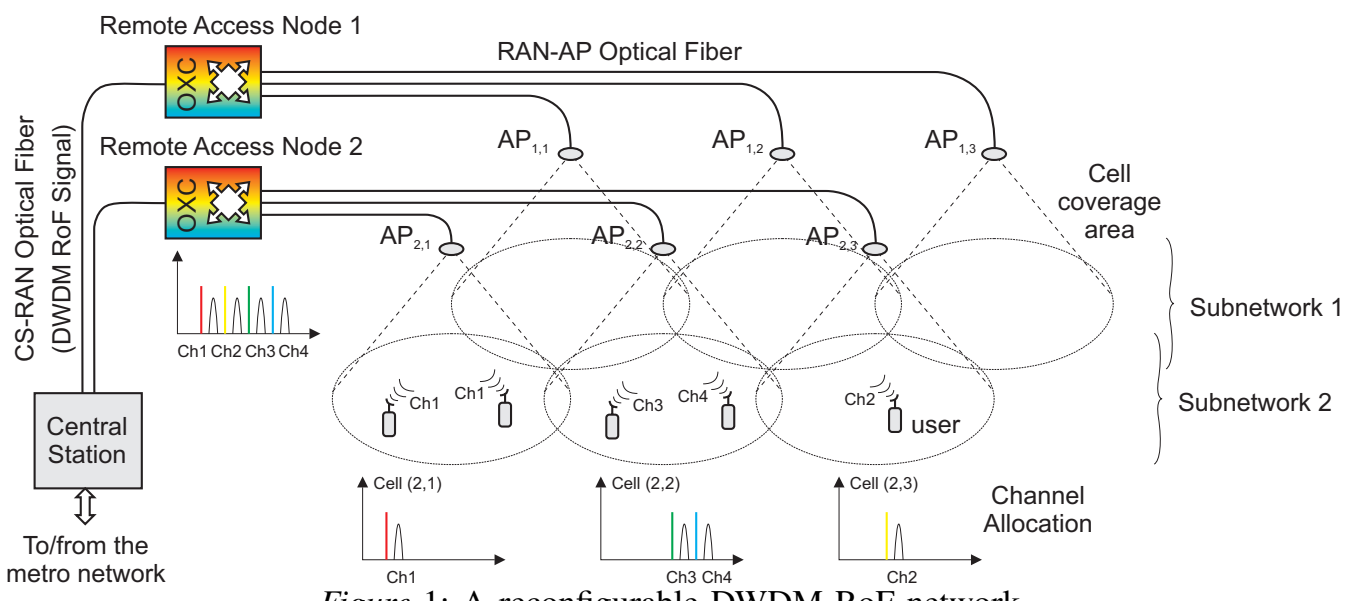

Figure 1: A reconfigurable DWDM RoF network.

Each RAN has some switching capabilities and is able to route given RoF channels towards selected APs through the RAN-AP links. An AP extracts the delivered RF signals from the RoF channels and broadcast them by means of radio antennas to the users, also called the demand points (DP), situated in the cell coverage area. Each user receives RF signals and reads data from the channel(s) to which it has granted access. The RoF channel allocation to the APs should be such that the RF signal interference in cells belonging to two adjacent subnetworks is avoided. Although in this paper we focus on the downstream service delivery, still the concept can be extended to a full-duplex connectivity, as discussed in [7].

\section{PROBLEM FORMULATION}

We consider the DWDM RoF network model as in Fig. 1. We use $\mathcal{N}$ to denote the set of subnetworks. Each subnetwork $n \in \mathcal{N}$ is identified with a RAN and the set $\mathcal{J}_{n}$ of APs. Let $\mathcal{I}_{n j}$ denote the set of DPs that are in the range of AP $j$ belonging to subnetwork $n$. In this paper, we assume that both the sets $\mathcal{I}_{n j}$, where $j \in \mathcal{J}_{n}, n \in \mathcal{N}$, and the sets $\mathcal{J}_{n}$, where $n \in \mathcal{N}$, are disjoint, i.e., each DP is in the range of only one AP and each AP belongs to a unique subnetwork. Let $n(j) \in \mathcal{N}$ identify the subnetwork the AP $j$ belongs to, i.e., $n(j)=\left\{n: n \in \mathcal{N}, j \in \mathcal{J}_{n}\right\}$. Let $\mathcal{V}=\bigcup_{n \in \mathcal{N}} \mathcal{J}_{n}$ and $\mathcal{U}=\bigcup_{n \in \mathcal{N}, j \in \mathcal{J}_{n}} \mathcal{I}_{n j}$ be, respectively, the set of all APs and the set of all DPs in the network.

Let $\mathcal{K}$ denote the set of RoF channels, each one of data capacity $b_{k}, k \in \mathcal{K}$. Each AP can have allocated a number of channels from $\mathcal{K}, c_{n j}$ at most, which allow to communicate with DPs. In the scope of subnetwork $n$, channel $k \in \mathcal{K}$ can be assigned only to one AP $j \in \mathcal{J}_{n}$ and such channel can be shared between different DPs belonging to $\mathcal{I}_{n j}$. Each DP communicates using only one radio channel, the one that it has assigned. The volume of traffic to be delivered to DP $i \in \mathcal{I}_{n j}$ is equal to $h_{i}$; for simplicity, we assume $h_{i} \leq b_{k}, k \in \mathcal{K}$.

Let $\mathcal{G}=(\mathcal{V}, \mathcal{E})$ denote the (unidirectional) interference graph; $\mathcal{V}$ is the set of all APs and $\mathcal{E}$ is the set of links, where link $\left(j^{-}, j^{+}\right) \in \mathcal{E}$ corresponds to a pair of interfering APs $j^{-}$and $j^{+}$. We assume that two interfering APs can not use the same channel $k \in \mathcal{K}$ at the same time.

To formulate the optimization problem we introduce a set of decision (binary) variables:

$x_{i} \in\{0,1\}$ - equal to 1 if DP $i \in \mathcal{U}$ is served, and equal to 0 otherwise,

$x_{i k} \in\{0,1\}$ - equal to 1 if DP $i \in \mathcal{U}$ operates on channel $k \in \mathcal{K}$, and equal to 0 otherwise,

$x_{n j k} \in\{0,1\}$ - equal to 1 if channel $k \in \mathcal{K}$ is allocated to $\operatorname{AP} j \in \mathcal{J}_{n}$ in subnetwork $n \in \mathcal{N}$, and equal to 0 otherwise.

The MSCA problem can be formulated as a 0-1 IP problem:

$$
\begin{aligned}
\text { maximize } & \sum_{i \in \mathcal{U}} h_{i} x_{i} \\
\text { subject to } & x_{i}-\sum_{k \in \mathcal{K}} x_{i k}=0, \quad \forall i \in \mathcal{U}, \\
& \sum_{j \in \mathcal{J}_{n}} x_{n j k} \leq 1, \quad \forall n \in \mathcal{N}, \forall k \in \mathcal{K}, \\
& \sum_{i \in \mathcal{I}_{n j}} h_{i} x_{i k}-x_{n j k} b_{k} \leq 0, \quad \forall n \in \mathcal{N}, \forall j \in \mathcal{J}_{n}, \forall k \in \mathcal{K}, \\
& \sum_{k \in \mathcal{K}} x_{n j k} \leq c_{n j}, \quad \forall n \in \mathcal{N}, \forall j \in \mathcal{J}_{n}, \\
& x_{n\left(j^{-}\right) j^{-}}+x_{n\left(j^{+}\right) j^{+} k} \leq 1, \quad \forall k \in \mathcal{K}, \forall\left(j^{-}, j^{+}\right) \in \mathcal{G}, \\
& x_{i}, y_{i}, x_{i k}, x_{n j k} \in\{0,1\}, \quad \forall n \in \mathcal{N}, \forall j \in \mathcal{J}_{n}, \forall i \in \mathcal{I}_{n j}, \forall k \in \mathcal{K} .
\end{aligned}
$$

Our problem objective (1a) is to maximize the volume of traffic delivered to DPs. Constraints (1b) are channel assignment constraints; indeed, a demand, whenever accepted, has assigned a channel. Constraints (1c) 


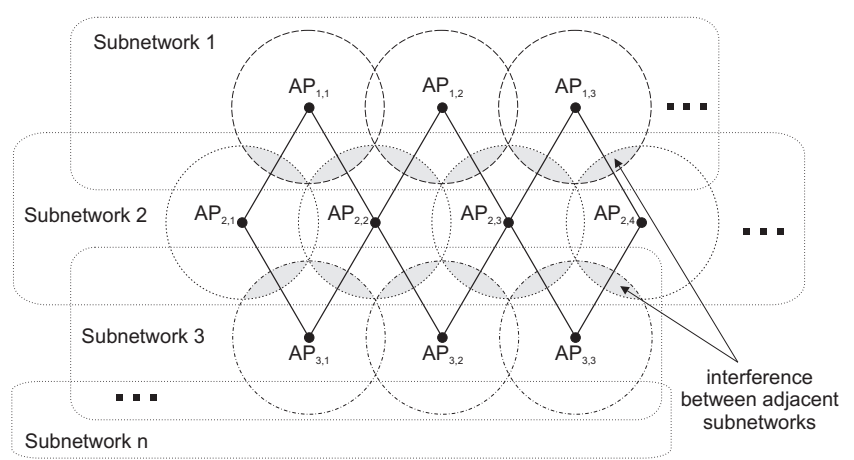

Figure 2: The case study RoF network and the channel interference graph.

say that in the scope of a subnetwork, each channel can be allocated only to one AP. Constraints (1d) and (1e) are, respectively, the channel capacity and the AP capacity constraints. Constraints (1f) are the interference constraints. Eventually, constraints $(1 \mathrm{~g})$ are the variable range constraints. It is worth nothing that the channel assignment problem can be further modified so that to focus on other objectives, e.g., the number of distinct channels used or fair access to the resources.

The special case of MSCA, in which $\left|\mathcal{J}_{n}\right|=1,\left|\mathcal{I}_{n j}\right|=c_{n j}, h_{i}=1$ for all $n \in \mathcal{N}, j \in \mathcal{J}_{n}, i \in \mathcal{I}_{n j}$, and $b_{k}=1$ for all $k \in \mathcal{K}$, is equivalent with the maximum service frequency assignment problem (Max-FAP) [8]. As a consequence, the MSCA optimization problem is $\mathcal{N} \mathcal{P}$-hard in general.

\section{NUMERICAL RESULTS}

To evaluate the problem complexity in this section we perform several numerical experiments. We consider the RoF access network topology presented in Fig. 2. The subnetworks are arranged side by side and in parallel. In each subnetwork, the APs are arranged in a straight line in such a way that the cells in the network form an equilaterally triangular grid. Due to the assumption about the orthogonality of RF signals in the subnetwork, the resulting interference graph forms a diamond-like grid form. It is worth nothing that the considered arrangement has been chosen arbitrarily and, depending on the application and environmental characteristics, other arrangements might be more suitable in particular deployment scenarios.

For the sake of simplicity of the presentation, we consider the same number of APs and RoF channels in each subnetwork, the same number of DPs in each cell, and the same traffic volume for each DP. Evaluated network scenarios differ in the number of subnetworks $|\mathcal{N}|$ and the number of APs $J$ per subnetwork (hence, in the total number of APs $|\mathcal{V}|=|\mathcal{N}| J)$, the number of RoF channels $|\mathcal{K}|$ per subnetwork, the allowed number of RoF channels $c$ that can be allocated to a single AP, the number of DPs $I$ per cell (hence, in the total number of DPs $|\mathcal{U}|=|\mathcal{V}| I$ ), and the traffic volume $h$ per DP (expressed as a fraction of the RoF channel capacity).

Regarding the network size, we rely on some indications given in [3] for a picocellurar network scenario. In particular, the authors observed that the increase of $J$ will result in both the decrease of $I$ and the decrease of $h$ in a given deployment area. We intend to maintain this principle and, in selected scenarios (namely, in scenarios $1-3$, and $4,7-8)$, we assume, hypothetically, that $(J, I, h) \in\{(1,64,1),(4,16,0.5),(16,4,0.1)\}$. Due to the limited scalability of switching elements in RAN, we consider the number of RoF channels $|\mathcal{K}| \leq 64$.

The MSCA optimization problem is solved by means of the IBM ILOG CPLEX v.12.1 solver [9]. The evaluation is performed on a Pentium M $2 \mathrm{GHz}$ computer. The performance is expressed in terms of the volume of delivered traffic and the computation time. In each case the optimality is achieved.

In Table I we present the MSCA optimization results. We can see that the computation times can range from a fraction of seconds, for networks with several APs (scenarios 1-4), to some thousands of seconds for larger networks (scenarios $14-16$ ). It is worth observing that the increase of the traffic volume results in the increase of the computation effort (compare scenarios 8 and 9), even for a reduced number of APs (scenario 8 vs. scenario 7). The increased traffic requirements involve the use of larger number of channels, what complicates the problem since the interference constraints have be satisfied for all the assigned channels. Besides, we can see that more channels available per subnetwork allows to find the solution in shorter time (scenario 13 vs. 9), supposedly because there is more opportunities to avoid interference. Finally, comparing scenarios 4,7 , and 8 , where the design objective is to have a fixed number of users served in a deployment area, we can see that the increase of the number of APs does not necessarily lead to the increase of the problem solution time. In fact, in scenario 8 , by increasing the number of APs (with respect to scenario 7), we decrease the number of users per cell and the relative (to the channel capacity) traffic demand per user what, in consequence, alleviates the problem. 


\begin{tabular}{|c|c|c|c|c|c|c|c|c||c|c|}
\hline \multicolumn{1}{|c|}{ Scenario } & \multicolumn{2}{|c|}{ Results } \\
\hline No & $|\mathcal{V}|$ & $|\mathcal{U}|$ & $|\mathcal{N}|$ & $J$ & $|\mathcal{K}|$ & $c$ & $I$ & $h$ & Delivered traffic & Time [sec] \\
\hline \hline 1 & 1 & 64 & 1 & 1 & 32 & 32 & 64 & 1 & $50 \%$ & 0.2 \\
\hline 2 & 4 & 64 & 1 & 4 & 32 & 32 & 16 & 0.5 & $100 \%$ & 0.25 \\
\hline 3 & 16 & 64 & 1 & 16 & 32 & 32 & 4 & 0.1 & $100 \%$ & 0.3 \\
\hline 4 & 16 & 1024 & 16 & 1 & 32 & 32 & 64 & 1 & $25 \%$ & 1.4 \\
\hline 5 & 64 & 320 & 8 & 8 & 16 & 16 & 5 & 0.5 & $80 \%$ & 11 \\
\hline 6 & 64 & 640 & 8 & 8 & 32 & 32 & 10 & 0.25 & $100 \%$ & 25 \\
\hline 7 & 64 & 1024 & 16 & 4 & 32 & 32 & 16 & 0.5 & $100 \%$ & 516 \\
\hline 8 & 256 & 1024 & 16 & 16 & 32 & 32 & 4 & 0.1 & $100 \%$ & 13 \\
\hline 9 & 256 & 1024 & 16 & 16 & 32 & 32 & 4 & 0.5 & $100 \%$ & 188 \\
\hline 10 & 256 & 1280 & 16 & 16 & 32 & 4 & 5 & 0.5 & $80 \%$ & 623 \\
\hline 11 & 256 & 1280 & 16 & 16 & 32 & 32 & 5 & 0.5 & $80 \%$ & 884 \\
\hline 13 & 256 & 1024 & 16 & 16 & 64 & 64 & 4 & 0.5 & $100 \%$ & 23 \\
\hline 14 & 256 & 2048 & 16 & 16 & 64 & 64 & 8 & 0.5 & $100 \%$ & 1161 \\
\hline 15 & 256 & 2560 & 16 & 16 & 32 & 4 & 10 & 0.25 & $80 \%$ & 5637 \\
\hline 16 & 256 & 2560 & 16 & 16 & 32 & 32 & 10 & 0.25 & $80 \%$ & 3857 \\
\hline
\end{tabular}

TABLE I: The MCA optimization results.

\section{CONCLUDING REMARKS}

In this paper, we address the problem of channel allocation in a DWDM RoF access network. The problem concerns the assignment of RoF resources, such as the optical frequencies and the radio frequencies, to the wireless access points so that to provide the users with the demanded bandwidth and avoid the channel interference. As a case study, we focus on the maximal service RoF channel allocation problem, which we formulate as a 0-1 integer programming problem. Although MSCA is $\mathcal{N} \mathcal{P}$-complete, still it can be resolved for a real-size network instances, of up to some hundreds of access points and users, in time of up to several hundreds of seconds. It makes the formulation applicable for off-line network design problems. On the other hand, in the light of dynamic network scenarios, e.g., when users are active temporally or move between cells, an on-line RoF channel allocation problem might require more time-effective methods, based either on heuristic algorithms or a straightened formulation of the problem. The variant of the MSCA problem we study in this paper assumes the orthogonality of RF signals in each subnetwork. A more general problem, where the interference of RF signals may occur between any pair of APs in the network, thus increasing the problem complexity, is left for further study.

\section{ACKNOWLEDGMENT}

This work has been supported by the Polish Ministry of Science and Higher Education under the contract 643/N-COST/2010/0.

\section{REFERENCES}

[1] N. Ghazisaidi, M. Maier, and Ch. M. Assi, "Fiber-wireless (FiWi) access networks: A survey", IEEE Comm. Mag., Feb. 2009.

[2] Ch. Lim et al., "Fiber-wireless networks and subsystem technologies", IEEE JLT, vol. 28, no. 4, Feb. 2010.

[3] M. Sauer, A. Kobyakov, and J. George, "Radio over fiber for picocellular network architectures", IEEE JLT, vol. 25, no. 11, Nov. 2007.

[4] G.-K. Chang et al., "Key technologies of WDM-PON for future converged optical broadband access networks", J. Opt. Commun. Netw., vol. 1, no. 4, Sep. 2009.

[5] T. Kuri et al., "Reconfigurable dense wavelength division multiplexing millimeter-wave-band radio-over-fiber access system technologies", IEEE JLT, vol. 28, no. 12, 2010.

[6] J. Vegas Olmos, T. Kuri, and K.-I. Kitayama, "Dynamic reconfigurable WDM 60-GHz millimeter waveband radio-over-fiber access network: Architectural considerations and experiment", IEEE JLT, vol. 25, no. 11, Nov. 2007.

[7] T. Sono et al., "Full-duplex 25-GHz spacing DWDM MM-wave-band radio-on-fiber system using a supercontinuum light source and arrayed waveguide-grating filters," in Proc. Int. Top. Meeting MWP, Oct. 2006, pp. 1-4, W15.

[8] A. Koster et al., "Models and solution techniques for frequency assignment problems", Annals of Operations Research, vol. 153, no. 1, pp. 79-129, Sep. 2007.

[9] "IBM ILOG CPLEX: High-performance mathematical programming engine.", http://www01.ibm.com/software/integration/optimization/cplex/, 2010. 